\title{
Pé diabético. Parte 1: Úlceras e Infecções*
}

\section{Diabetic Foot. Part 1: Ulcers and Infections}

\author{
Ricardo Cardenuto Ferreira ${ }^{1}$ \\ ${ }^{1}$ Grupo de Cirurgia do Pé e Tornozelo, Departamento de Ortopedia e \\ Traumatologia da Santa Casa de Misericórdia de São Paulo, Sâo Paulo \\ SP, Brasil
}

Endereço para correspondência Ricardo Cardenuto Ferreira, MD, Rua Barata Ribeiro, 380, Cj 64, 6 . Andar, Bela Vista, SP, 01308-000, Brasil (e-mail: ricardocardenuto@gmail.com).

Rev Bras Ortop 2020;55(4):389-396.

\begin{abstract}
Resumo
Palavras-chave

- diabetes

- pé

- úlcera

- infecção

- amputação

Abstract

Keywords

- diabetes

- foot

- ulcer

- infection

- amputation

As úlceras e infecções são complicações comuns nos pés dos pacientes diabéticos que já se encontram na fase tardia desta doença sistêmica que se tornou uma verdadeira epidemia do mundo moderno. Neste grupo específico de pacientes, são as infecções que constituem o principal fator envolvido na sequência de eventos que resultam na amputação do membro inferior. A neuropatia periférica (NC) constitui fator determinante na perda da sensibilidade protetora dos pés na fase tardia da doença e, por sua vez, favorece o desenvolvimento das úlceras plantares de pressão (UPP) e a destruição osteoarticular causado pela neuroartropatia de Charcot (NC). A obesidade, a doença arterial periférica (DAP) e a deficiência no sistema imunológico devida aos distúrbios metabólicos do diabetes desempenham papel adicional importante na morbidade desta doença, principalmente no que se refere à amputação dos membros inferiores. É importante para o médico ortopedista compreender que para tentar evitar estas complicações que costumam resultar na amputação da extremidade é necessário estabelecer estratégias de prevenção direcionadas, principalmente, para a educação do paciente diabético e também para medidas protetoras profiláticas.

Diabetes is a systemic disease that has achieved epidemic proportions in modern society. Ulcers and infections are common complications in the feet of patients with advanced stages of the disease, and are the main cause of amputation of the lower limb. Peripheral neuropathy is the primary cause of loss of the protective sensation of the feet and frequently leads to plantar pressure ulcers and osteoarticular disruption, which in turn develops into Charcot neuropathy (CN). Common co-factors that add to the morbidity of the disease and the risk of amputation in this population are obesity, peripheral arterial disease, immune and metabolic disorders. Orthopedic surgeons must be aware that the early detection and prevention of these comorbidities, through diligent medical care and patient education, can avoid these amputations.
\end{abstract}

\footnotetext{
Trabalho desenvolvido no Grupo de Cirurgia do Pé e Tornozelo, Departamento de Ortopedia e Traumatologia da Santa Casa de Misericórdia de São Paulo, São Paulo, SP, Brasil.
}

recebido

25 de Junho de 2019

aceito

13 de Setembro de 2019
DOI https://doi.org/

10.1055/s-0039-3402462. ISSN 0102-3616.
Copyright $\odot 2020$ by Sociedade Brasileira License terms de Ortopedia e Traumatologia. Published by Thieme Revinter Publicações Ltda, Rio de Janeiro, Brazil 


\section{Introdução}

O diabetes mellitus (DM) é uma grave doença sistêmica cuja incidência vem aumentando em paralelo ao aumento nos índices de obesidade da população mundial. ${ }^{1}$ Uma perspectiva sombria é estimada para o ano de 2040, quando se acredita que haverá 642 milhões de diabéticos no mundo, o que equivale a dizer que $\sim 10 \%$ de toda população do planeta será diabética. ${ }^{2}$

O acometimento dos pés nos pacientes diabéticos está associado com um processo crônico que cria condições propícias para o aparecimento da úlcera plantar no pé (UPP). ${ }^{3}$ Entre estes fatores desencadeantes, destacam-se: 1 ) a neuropatia periférica (NP) que provoca perda na sensibilidade protetora dos pés; 2) a doença arterial periférica (DAP); e 3) as alterações biomecânicas provocadas pela destruição osteoarticular e deformidades decorrentes da neuroartropatia de Charcot (NC), responsáveis por alterar as pressões de apoio na planta do pé. ${ }^{3}$

A prevenção e o tratamento da UPP constitui uma das maiores preocupações no cuidado com os pacientes diabéticos. ${ }^{4}$ Mesmo quando todos os cuidados preventivos são adequadamente adotados, a incidência anual de aparecimento de UPP atinge um índice aproximado de $2,2 \%{ }^{5}$ Infecções ocorrem em até $58 \%$ dos pacientes que apresentam uma nova UPP e, em decorrência, $\sim 5 \%$ destes pacientes sofrerão uma amputação maior no prazo de até 1 ano. ${ }^{6,7}$ Pacientes diabéticos que desenvolvem lesões nos pés estão sujeitos à elevada incidência de morte prematura diretamente associada ao alto risco de sofrer uma amputação maior em pelo menos um dos membros inferiores ao longo da vida. ${ }^{8} \mathrm{O}$ índice de mortalidade num prazo de até 5 anos após o surgimento de uma UPP chega a $45 \%$ nos pacientes que apresentam úlcera de causa predominantemente neuropática, e a $55 \%$ naqueles com úlcera de causa preponderante no componente isquêmico. ${ }^{9}$

De acordo com o sistema de saúde norte-americano, é estimado que $\sim 20 \%$ a $40 \%$ do montante de recursos disponíveis para o tratamento dos pacientes diabéticos são gastos no tratamento das complicações das lesões que afetam os pés. ${ }^{10}$ Uma perspectiva do grande impacto do custo potencialmente envolvido no tratamento da UPP complicada com infecção no paciente diabético é oferecida por estudos que estimam gastos que variam de U\$ 3.000 (Tanzânia) até U\$ 188.000 (Estados Unidos). ${ }^{11}$

O objetivo da presente revisão é salientar os principais aspectos da fisiopatologia e do tratamento das complicações do diabetes que afetam os pés, destacando-se as úlceras e as infecções secundárias. Salientaremos a importância da história clínica e do exame físico no correto diagnóstico destas lesões, com ênfase no estadiamento de acordo com a gravidade das diversas situações clínicas que se apresentam na rotina do atendimento destes pacientes. Acreditamos ser de alta importância fornecer informações capazes de permitir ao médico ortopedista realizar escolhas terapêuticas adequadas para tentar prevenir e, se possível, evitar a amputação da extremidade nos pacientes diabéticos.

\section{Fisiopatologia}

A etiologia das lesões no pé do paciente diabético é multifatorial, e inclui complicações da neuropatia, vasculopatia, imunodeficiência e descontrole da glicemia. ${ }^{12}$ A neuropatia dos nervos periféricos resulta na perda da sensibilidade, da capacidade motora (principalmente da musculatura intrínseca do pé) e no déficit autonômico. Além disso, é sem dúvida nenhuma a principal causa envolvida no surgimento de úlceras nos pés e, quase invariavelmente, está presente nos casos de $\mathrm{NC}^{3,12,13} \mathrm{~A}$ neuropatia motora provoca mudanças estruturais no pé devido, em parte, ao desequilíbrio muscular e fraqueza da musculatura intrínseca. As deformidades mais frequentemente desencadeadas pela neuropatia motora são: dedos em garra, dedos em martelo, proeminência plantar das cabeças dos metatarsos e pé cavo. ${ }^{3,13,14}$ Estas deformidades alteraram os padrões da pressão plantar durante a marcha e tornam os pés insensíveis ainda mais susceptíveis às úlceras de pressão., 313

Aproximadamente $50 \%$ dos pacientes diabéticos apresentam algum grau de DAP ${ }^{15}$ e, devido ao processo neuroisquêmico, esta contribui diretamente com o desenvolvimento da neuropatia e consequentes complicações nos pés. ${ }^{13,16}$ Nos pacientes diabéticos afetados pela NC, a presença de DAP em estágio avançado é menos comum do que nos pacientes que apresentam apenas úlcera de pressão. ${ }^{17} \mathrm{~A}$ imunodeficiência envolvendo tanto a habilidade fagocitária dos leucócitos quanto sua capacidade de produzir anticorpos (linfócitos T) é bem reconhecida nos pacientes diabéticos, contribuindo diretamente para a baixa resposta imunológica no combate das infecções. ${ }^{18,19}$ Tanto a DAP quanto a imunodeficiência não contribuem diretamente para a formação das úlceras, mas atuam como fatores de risco, aumentando a chance de complicação nos pacientes diabéticos com neuropatia. ${ }^{12}$

\section{Avaliação Inicial}

\section{História Clínica e Exame Físico}

Uma história clínica detalhada é crucial no adequado manejo das complicações relacionadas aos pés dos pacientes diabéticos. É extremamente importante coletar adequadamente informações precisas referentes ao tempo de duração da doença, insulino-dependência, comorbidades pré-existentes, cirurgias prévias, histórico familiar, histórico pessoal (tabagismo, alcoolismo, dependência de drogas ilícitas, disponibilidade de apoio e assistência familiar), e medicações atualmente em uso. ${ }^{5,13}$

No exame físico, é essencial verificar a presença de qualquer déficit na sensibilidade protetora dos pés utilizando o teste do monofilamento 5.07 de Semmes-Wienstein. ${ }^{20}$ A pele deve ser examinada na busca dos sinais da neuropatia autonômica, principalmente no que se refere ao ressecamento e a presença de fissuras cutâneas. Evidências da neuropatia motora podem ser observadas principalmente quando existe desequilíbrio na musculatura intrínseca do pé causando deformidade em garra ou martelo dos dedos e, consequentemente, propiciando a proeminência plantar das cabeças dos metatarsos. ${ }^{14}$ É importante pesquisar a presença de tensão excessiva na musculatura posterior da perna formado pelo complexo sóleo-gastrocnêmio que pode estar 
encurtado, provocando por sua vez restrição no arco de movimento do tornozelo ou mesmo equino residual, responsável pela geração de áreas de hiperpressão na região plantar do antepé. Na inspeção estática, é vital verificar se existem sinais de calosidades ou úlceras localizadas sob as áreas aonde se identificam proeminências ósseas na região plantar do antepé. Com o paciente posicionado em pé, é importante pesquisar a presença de edema, avaliar o adequado alinhamento dos pés e tornozelos, além de buscar identificar sinais de instabilidade na região do tornozelo ou envolvendo todo o retropé. Durante a marcha, é possível verificar a exacerbação de possível instabilidade nestas articulações. ${ }^{5,13}$

\section{Testes Vasculares}

Os pacientes diabéticos apresentam elevado risco de desenvolver doença vascular que compromete a macro e a microcirculação. ${ }^{5,13}$ Na doença macrovascular, ocorre oclusão progressiva tanto da artéria femoral profunda quanto do segmento trifurcado infra poplíteo (artérias tibial anterior, posterior e fibular). ${ }^{15}$ Desta forma, é importante durante a avaliação clínica sempre realizar a palpação dos pulsos poplíteo, tibial posterior e dorsal do pé. Quando é possível palpar os pulsos tanto da artéria tibial posterior quanto da dorsal do pé, é improvável que haja real necessidade de algum tipo de intervenção vascular, uma vez que a circulação do pé é adequada. ${ }^{14}$ Por outro lado, a ausência de pulsos palpáveis no pé possui sensitividade de $\sim$ $70 \%$ no diagnóstico da doença vascular periférica e, quando isto for constatado, é recomendável solicitar avaliação especializada de um cirurgião vascular. ${ }^{14} \mathrm{Na}$ doença microvascular, tipicamente ocorre o comprometimento da microcirculação que afeta principalmente os seguintes órgãos: 1) vasos da retina podendo provocar amaurose; 2) vasos glomerulares com consequente comprometimento da função renal, podendo resultar em falência completa deste órgão; e 3) vasos que nutrem os nervos periféricos (vasa nervorum) causando degeneração progressiva e neuropatia sensitivo-motora-autonômica. ${ }^{14,21}$ Durante o exame clínico, é importante identificar sinais aparentes de doença arterial periférica por meio da percepção de pele fria durante a palpação do pé, além de observar a diminuição ou ausência dos pelos, vermelhidão cutânea e pele brilhante., ${ }^{5,13}$

A princípio, a avaliação vascular não invasiva deve acessar a presença ou não do fluxo sanguíneo, sua velocidade e formato da onda por meio de ultrassom doppler. ${ }^{13}$ Testes anormais são indicativos da presença de doença macrovascular e identificam a necessidade de solicitar consulta com um cirurgião vascular para uma avaliação mais detalhada. ${ }^{13}$ Para avaliar possíveis alterações na microcirculação, é de grande valia utilizar o aparelho de oximetria para mensurar a pressão transcutânea de oxigênio na extremidade. De acordo com o grau de oxigenação tecidual aferido, é possível estimar o grau de comprometimento da microcirculação e o potencial de cicatrização local da UPP. ${ }^{13}$ Quaisquer sinais evidentes de isquemia requerem consulta imediata de um médico especialista no tratamento endovascular e, frequentemente, é necessária realização de angiografia para avaliar potencial intervenção para desobstruir uma artéria que eventualmente esteja obstruída. ${ }^{13}$ A revascularização da extremidade pode restaurar a circulação macrovascular; entretanto, alterações no sistema de circulação microvascular irão persistir e podem, frequentemente, provocar impacto negativo na cicatrização das feridas na pele ou nas incisões cirúrgicas. ${ }^{13}$

\section{Úlceras no Pé}

As UPPs nos pacientes diabéticos ocorrem em 15\% daqueles que já apresentam neuropatia periférica com perda da sensibilidade protetora. $^{22}$ Frequentemente resultam de trauma repetitivo ou de padrões de pressão excessiva que atuam numa extremidade cuja sensibilidade encontra-se muito diminuída ou ausente. Quando se detecta a presença de úlcera, é necessário interrogar o paciente sobre o tempo de duração e o estado de progressão do tamanho da lesão. A persistência de uma úlcera no mesmo local por $>30$ dias está associada com significativo aumento no risco de infecção que pode, eventualmente, progredir para osteomielite. ${ }^{23}$ Além disso, é muito importante identificar a exata localização da úlcera, mensurar precisamente seu diâmetro e profundidade, além de avaliar a presença ou não de sensibilidade protetora e de sangramento ativo nas margens da ferida. Úlceras cujo tamanho excede $2 \mathrm{~cm}^{2}$ estão mais propensas à osteomielite. ${ }^{23,24}$

\section{Classificação das Úlceras}

O Sistema de Classificação da Universidade do Texas é altamente eficiente como fator preditivo da cicatrização das úlceras e foi eleito como o sistema de classificação padrão recomendado pelos especialistas. ${ }^{25,26}$ Ele foca na avaliação da profundidade da úlcera, na presença ou não de abscesso, osteomielite, pioartrite e gangrena, além de documentar a presença de isquemia na extremidade. ${ }^{3}$ (- Tabela $\mathbf{1}$ )

Tabela 1 Classificação da Universidade do Texas para feridas no pé

\begin{tabular}{|l|l|l|l|l|}
\hline & Grau 0 & Grau 1 & Grau 2 & Grau 3 \\
\hline Estágio A & $\begin{array}{l}\text { Pele epitelizada } \\
\text { (pré- ou pós-úlcera) }\end{array}$ & $\begin{array}{l}\text { Ferida superficial sem } \\
\text { envolvimento de tendão, } \\
\text { cápsula articular, ou osso }\end{array}$ & $\begin{array}{l}\text { Ferida profunda atinge } \\
\text { plano do tendão ou } \\
\text { cápsula articular }\end{array}$ & $\begin{array}{l}\text { Ferida profunda atinge } \\
\text { plano da articulação } \\
\text { ou do osso }\end{array}$ \\
\hline Estágio B & Com infecção & Com infecção & Com infecção & Com infecção \\
\hline Estágio C & Com isquemia & Com isquemia & Com isquemia & Com isquemia \\
\hline Estágio D & Infecção e isquemia & Infecção e isquemia & Infecção e isquemia & Infecção e isquemia \\
\hline
\end{tabular}




\section{Tratamento das Úlceras}

O tratamento inicial da UPP inclui debridamento local, retirada da carga de apoio sobre o pé, e curativos frequentes. 0 debridamento local não requer nenhum tipo de anestesia devido à ausência de sensibilidade causada pela neuropatia periférica, e é capaz de transformar uma ferida crônica numa ferida aguda na medida em que remove o tecido necrótico e diminui o número de bactérias que formam o biofilme ao redor da úlcera, criando um ambiente favorável para a formação de tecido de granulação saudável. ${ }^{13} \mathrm{~A}$ retirada da carga impedindo o apoio na planta do pé, combinada com a criação de um ambiente sangrante na base da úlcera, é essencial para a cicatrização das úlceras neuropáticas. A troca frequente do curativo mantendo a ferida limpa aparentemente é suficiente para promover a cicatrização desde que a pressão local tenha sido drasticamente reduzida. Não existe evidência suficiente para apoiar qualquer tipo de recomendação no que se refere ao uso de medicações tópicas, ou mesmo curativos comercialmente desenvolvidos, produtos estes que normalmente possuem alto custo e alegam possível benefício de acelerar a cicatrização das UPP. ${ }^{13}$

Modificações nas condições do paciente são cruciais para facilitar a cicatrização das UPPs e incluem: controle glicêmico adequado, otimização do estado nutricional, interrupção total do tabagismo, e melhora na circulação da extremidade. ${ }^{13} 0$ prognóstico favorável na cicatrização da UPP pode ser mensurado verificando se ocorreu redução de pelo menos $50 \%$ no diâmetro da úlcera após 4 semanas empregando tratamento adequado por meio de cuidados locais com a ferida e eliminação da carga de apoio na extremidade afetada; caso contrário, o potencial de cicatrização espontânea da ferida é baixo. ${ }^{27,28}$

\section{Retirada da Carga}

Um dos componentes mais importantes no tratamento da UPP é a retirada da carga através das seguintes alternativas: 1) utilização de sandálias terapêuticas com solado elevado em cunha; 2) botas removíveis; 3) andadores; 4) órteses confeccionadas sob medida; 5) gesso de contato total (GCT).

O GCT ou órteses confeccionadas sob medida especialmente desenhadas para reduzir a pressão plantar da UPP continuam sendo o procedimento padrão de tratamento das UPPs. ${ }^{27-29}$ A superioridade do GCT sobre as órteses removíveis no tratamento da UPP reside no fato da aderência do paciente ao tratamento, uma vez que estudos indicam que $o$ tempo médio de utilização das órteses removíveis restringese à apenas $28 \%$ de todas as atividades diárias. ${ }^{30}$

A cirurgia para alongamento percutâneo do tendão de Aquiles pode ser indicada em associação ao tratamento das úlceras plantares do antepé com o GCT quando existe restrição na dorsiflexão do tornozelo (não é possível dorsifletir esta articulação além de $90^{\circ}$ ). $O$ aumento na capacidade de dorsifletir o tornozelo propiciado pelo alongamento do tendão de Aquiles costuma auxiliar na diminuição da pressão plantar no antepé, acelerar a cicatrização das úlceras plantares e diminuir a incidência de recorrência destas lesões em médio prazo. ${ }^{31}$

\section{Modalidades Avançadas para Cicatrização das Úlceras} Mais recentemente, a oxigenoterapia hiperbárica (OTHB) e a terapia utilizando pressão negativa (TPN) têm sido advogadas como modalidades avançadas para acelerar a cicatrização das feridas. Existe um debate considerável sobre a real eficácia destas propostas de tratamento. Estudos prospectivos randomizados controlados e duplo-cego são inconclusivos em relação ao efeito da OTHB ou TPN na redução nos índices de amputações quando comparados com o tratamento realizado apenas com cuidados locais da ferida. ${ }^{32-34}$

\section{Recidiva das Úlceras}

A educação dos pacientes desempenha papel fundamental na prevenção da recidiva da UPP. À medida que os pacientes conseguem compreender o mecanismo que provoca as lesões, ele pode participar mais ativamente e aderir às estratégias voltadas a evitar o surgimento de novas úlceras. A despeito da maior participação dos pacientes na prevenção, a taxa de recidiva é alta e atinge níveis superiores a $40 \%{ }^{35-37}$ Esta elevada prevalência se deve justamente ao fato da NP e da DAP persistirem como os verdadeiros fatores diretamente envolvidos na fisiopatologia destas lesões. ${ }^{13}$ Devido ao seu curso marcado pelas recidivas frequentes, a ulceração recorrente é altamente propensa a desenvolver complicações graves que envolvem infecção profunda, formação de abscesso e osteomielite. $^{38,39} \mathrm{Em}$ decorrência disto, o risco estimado de ser necessária amputação da extremidade, em algum momento do curso desta afecção, varia de 71 a $85 \%$ dos casos. ${ }^{40,41}$

\section{Infecções no Pé}

Aproximadamente 50\% das UPPs sofrem infecção secundária por contiguidade, causando profundo impacto negativo na qualidade de vida do paciente. ${ }^{6,42}$ Os principais fatores de risco associados à infecção das UPPs são: 1) lesões ulceradas profundas; 2) úlceras presentes por $>30$ dias; 3 ) histórico prévio de úlceras recorrentes; 4) lesões de etiologia traumática; 5) presença concomitante de DAP. ${ }^{23}$

Ao exame clínico, é importante ressaltar que o paciente diabético pode não manifestar sinais e sintomas típicos de uma infecção grave (mal estar geral, torpor, náusea, anorexia e febre) em decorrência da sua deficiente resposta imunoleucocitária. ${ }^{43} \mathrm{O}$ sinal mais precoce da presença de uma infecção grave é a hiperglicemia que não regride mesmo quando a dosagem de insulina prescrita é significativamente aumentada. ${ }^{5,13}$ São fundamentais, neste momento, realizar inspeção detalhada da úlcera verificando os seguintes aspectos: 1 ) sua extensão (diâmetro $>2 \mathrm{~cm}$ constitui sinal de alerta); 2) sua profundidade (introduzir um instrumento através da ferida e perceber que este toca o osso subjacente é indício de gravidade e constitui fator preditivo de osteomielite com índice de acerto entre 53 e 89\%); 3) seu odor (quando fétido é sugestivo da presença de abscesso profundo); 4) suas margens; 5) a presença de drenagem (saída de secreção amarelada turva e densa denota presença de pus). ${ }^{23,24,36,44-48}$ É sempre recomendável realizar a elevação da extremidade por $\sim 5$ a 10 minutos para determinar se o eritema eventualmente presente é decorrente de um processo infeccioso (o eritema não regride) ou da $\mathrm{NC}$ (o eritema regride). $3,49,50$

Testes laboratoriais simples, tais como: 1) a presença de aumento na velocidade de hemossedimentação (VHS); 2) 
hiperglicemia; e 3) leucocitose, podem auxiliar no diagnóstico de uma infecção ativa. Radiografias simples com incidências convencionais para o pé e tornozelo podem mostrar imagens com sinais de ruptura na cortical do osso subjacente à região correspondente da UPP. Estas alterações são altamente sugestivas da presença de osteomielite; entretanto, surgem mais tardiamente, só se tornando visíveis após 10 a 20 dias desde o início da infecção óssea. ${ }^{51,52}$

Uma vez diagnosticada a infecção, é necessário admitir o paciente no hospital para tratamento imediato. Testes laboratoriais necessários para monitorar a evolução do estado clínico do paciente durante o tratamento devem ser solicitados e repetidos com certa regularidade. Destacam-se, entre eles: 1 ) hemograma completo; 2) VHS; 3 ) proteína (-reativa; 4) dosagem de albumina; 5) dosagem glicêmica; 6) provas de função renal e hepática (dosagem sérica de uréia, creatinina, transaminase glutâmico-oxalacética [TGO], transaminase glutâmico-pirúvica [TGP]). ${ }^{13}$ É recomendável eleger um médico clínico para acompanhar e monitorar as funções metabólicas do paciente durante todo o seu período de internação para tratamento da infecção.

Para avaliar a gravidade da situação, é importante dimensionar a real extensão do envolvimento inicial tanto das partes moles quanto dos ossos e articulações., ${ }^{3,49}$ Radiografias simples podem ser bastante úteis, e é necessário que sejam estudadas com atenção na busca dos seguintes sinais: 1) erosão do osso cortical; 2) reação periosteal; 3) imagens sugestivas da presença de gás nos tecidos moles (frequentemente produzido por germes anaeróbios); 4) imagens radiopacas sugestivas da presença de possíveis corpos estranhos provenientes de ferimento prévio não reconhecido pelo próprio paciente. ${ }^{5,21,51,52}$ Cintilografia óssea e ressonância nuclear magnética podem ser úteis e auxiliar na avaliação do diagnóstico de osteomielite. ${ }^{23,51-55}$

Uma vez identificada presença de infecção, é necessário coletar amostras de tecido profundo, preferencialmente tecido ósseo, a partir da úlcera, e enviá-lo para realização de cultura tanto de bactérias aeróbias quanto anaeróbias. ${ }^{53}$ É importante identificar o germe infectante para selecionar adequadamente $o$ antibiótico mais apropriado. ${ }^{26,33}$

\section{Tratamento das Infeç̧ões}

O tratamento das infecções no pé é ditado pela gravidade do quadro. Infecções superficiais devem ser tratadas com debridamento cirúrgico para remoção de todo tecido necrótico, curativo úmido e medidas para impedir o apoio da carga no pé. Além disso, é necessário agregar aos cuidados mencionados a prescrição de antibióticos empíricos ministrados por via oral, além de acompanhamento ambulatorial com visitas frequentes a fim de supervisionar de perto a evolução do quadro clínico. ${ }^{3,13,49}$ A duração do tratamento com antibiótico é controverso, mas este deve ser mantido até a resolução da infecção. ${ }^{25}$

Algumas infecções moderadas e todas as infecções profundas e graves necessitam de internação hospitalar imediata para início do tratamento o mais rápido possível a fim de reduzir o risco de amputação. ${ }^{46,56}$ Nos casos graves, é mandatório realizar intervenção cirúrgica precoce voltada para drenar os abscessos profundos e remover, por meio de cuidadoso debridamento, tanto os tecidos moles desvitalizados quanto todo osso infectado e necrótico. Ênfase deve ser dada na recomendação para deixar a ferida operatória completamente aberta para permitir drenagem contínua e evitar a coleção de novo abscesso. ${ }^{46,56}$ (-Figura 1) Frequentemente, são necessárias múltiplas

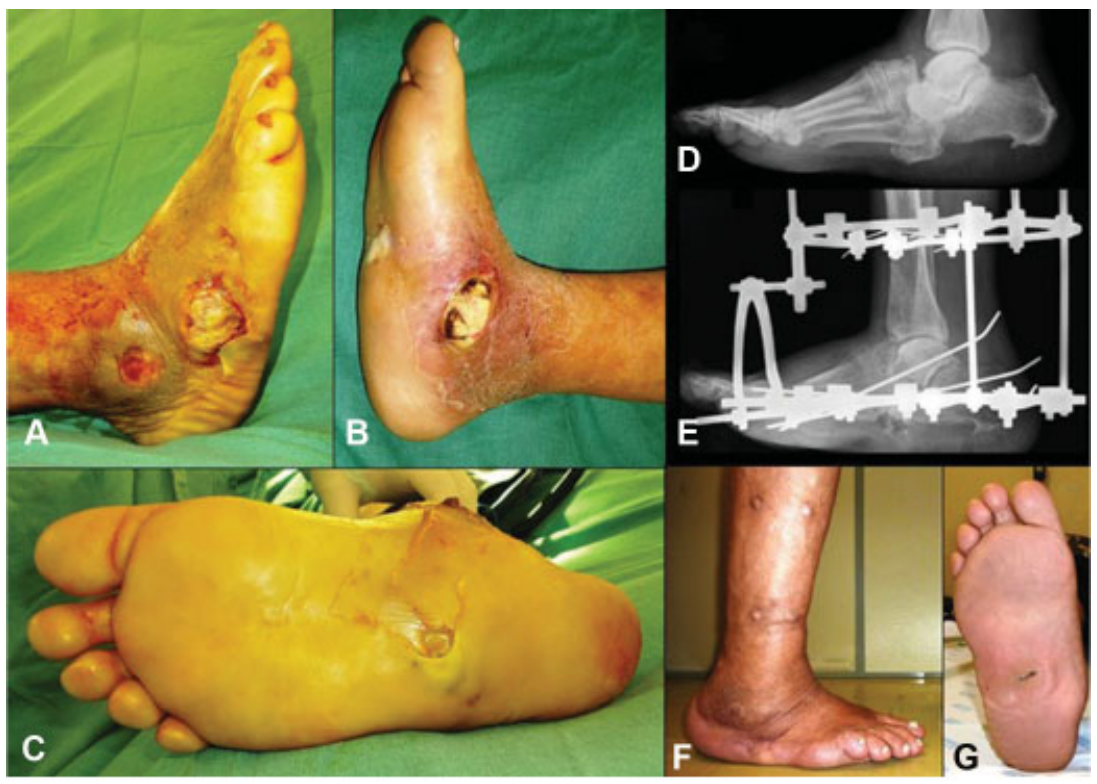

Fig. 1 Imagens lateral (A), medial (B) e plantar (C) do pé direito, mostrando a presença de múltiplas úlceras infectadas com necrose tecidual associada. Na imagem radiográfica pré-operatória, realizada na incidência lateral do pé, nota-se luxação talonavicular e saliência plantar do osso cubóide no mediopé (D). Após o debridamento cirúrgico e remoção do osso cubóide luxado, a imagem radiográfica na incidência lateral do pé mostra a estabilização da extremidade com o fixador externo circular (E). Seis meses após o tratamento foi possível evitar a amputação da extremidade e o pé encontra-se alinhado e livre da infecção e das úlceras (F e G). 


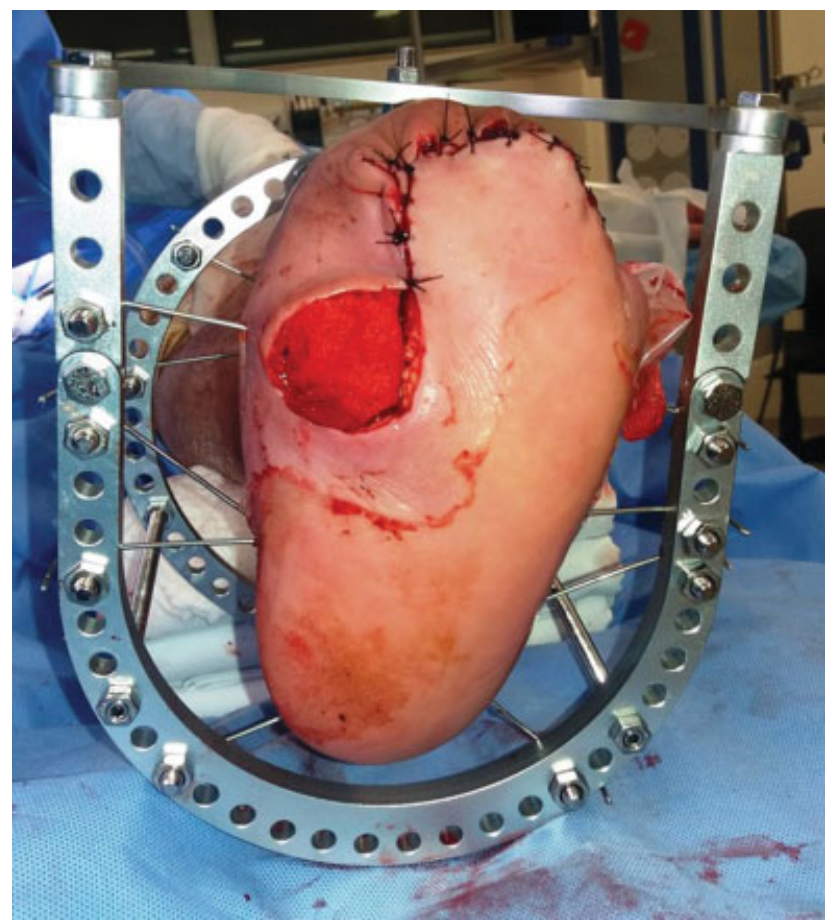

Fig. 2 Vista plantar do pé esquerdo após cirurgia para debridamento de úlcera plantar infectada seguida de drenagem de abscesso, remoção do tecido desvitalizado e amputação parcial transmetatarsal. A área correspondente à úlcera foi deixada aberta e recoberta com curativo oclusivo com gaze umedecida por soro fisiológico. A extremidade foi estabilizada com fixador externo circular e o paciente mantido internado para antibioticoterapia endovenosa sistêmica de largo espectro.

intervenções programadas sequencialmente num curto intervalo de tempo ( $\sim 48$ horas) para certificar-se que todo o tecido necrótico tenha sido completamente removido e tenha restado apenas tecido viável não infectado no leito do debridamento, o bem conhecido tecido de granulação (-Figura 2).

Antibioticoterapia endovenosa é sempre necessária no tratamento das infecções graves e sua duração depende da extensão da infecção. ${ }^{25}$ Culturas do tecido ósseo coletado na cirurgia de debridamento são importantes para direcionar o tratamento com antibiótico específico. ${ }^{33,55}$ Pode ser necessário consultar um especialista em infectologia para auxiliar na seleção e monitorização dos antibióticos, mesmo porque a combinação de bactérias aeróbias e anaeróbias nas infecções profundas costuma ser a regra e, frequentemente, é necessária associação antibiótica de drogas com potencial nefrotóxico e hepatotóxico utilizadas por período prolongado (usualmente de 6 a 12 semanas). ${ }^{55}$

\section{Amputações}

Mesmo na vigência do tratamento adequado imediato, as infecções no pé do paciente diabético podem ser difíceis de serem controladas, e a possibilidade de amputação sempre está presente e deve ser discutida previamente com o paciente. ${ }^{5,13,49}$ Estudos demonstram que o risco de amputação ultrapassa $20 \%$ dos casos de infecção moderada ou grave. ${ }^{44,57,58}$ Os pacientes diabéticos que desenvolvem infecção no pé apresentam risco 56 vezes maior de serem hospitalizados e 154 vezes maior de necessitarem de algum tipo de amputação do que aqueles que não sofrem nenhuma infecção. ${ }^{23}$ Os níveis de amputação mais frequentemente empregados envolvem o antepé, o mediopé, Syme, transtibial (abaixo do joelho), e transfemoral (acima do joelho). ${ }^{5,13,49}$ O cirurgião deve considerar os fatores e requerimentos específicos de cada paciente antes de decidir qual o nível mais apropriado para a amputação, objetivando sua reintegração familiar e social, além de permitir a possibilidade de recuperação funcional compatível com suas condições e capacidades funcionais. ${ }^{5,13,49}$ Apesar da meta do cirurgião em preservar o maior comprimento possível da extremidade no esforço de reduzir o gasto energético necessário para a deambulação após uma amputação, é necessário avaliar a habilidade de cicatrização do paciente antes de realizar a cirurgia. ${ }^{13}$ Vale a pena ressaltar que a morbidade e a mortalidade após a realização das amputações maiores (transtibial ou transfemoral) é elevada, atingindo índice de $29 \%$ nos 2 primeiros anos após a cirurgia. ${ }^{13}$ Diabéticos com insuficiência renal crônica que necessitam de diálise são particularmente vulneráveis a amputações maiores, atingindo taxa de mortalidade de $52 \%$ nos 2 anos seguintes à cirurgia. ${ }^{13}$ Além disso, $\sim 10 \%$ dos pacientes amputados necessitam de amputação transtibial contralateral. ${ }^{13}$ Apesar dos problemas relacionados à amputação, ela proporciona uma chance melhor de recuperação quando comparado a múltiplas tentativas de salvamento num paciente doente. ${ }^{5,49}$ Em alguns pacientes selecionados, a amputação de uma extremidade gravemente comprometida pode melhorar significativamente a qualidade de vida, melhorando inclusive sua capacidade física e permitindo manter a deambulação independente com uso de prótese do membro inferior. ${ }^{59}$

\section{Prevenção}

Os diferentes problemas que podem afetar os pés nos pacientes diabéticos muitas vezes se apresentam inicialmente como sinais ocultos, o que dificulta o diagnóstico imediato. É necessário e fundamental que o médico tenha elevado grau de suspeita e realize vigilância constante e altamente apurada para detectar precocemente situações potencialmente graves. Podemos afirmar, sem sombra de dúvida, que no paciente diabético o diagnóstico precoce e acurado das complicações é essencial para obter sucesso no tratamento.

A prevenção deve ser o foco principal de atenção para tentar evitar a sequência de eventos que podem desencadear a amputação da extremidade. ${ }^{5,13,49}$ Evitar o desenvolvimento da UPPs, e tratar as pré-existentes para tentar impedir que as mesmas se infectem, é tarefa árdua que exige máxima atenção e participação do paciente, de seus familiares e dos profissionais de saúde encabeçados pelo médico. Desempenham papel crucial na prevenção da UPP as seguintes ações: 1) a realização de programas de educação do paciente; 2) o incentivo à utilização de calçados protetores e de palmilhas moldadas confeccionadas com material macio (indicadas 
para acomodar deformidades pré-existentes e também reduzir o atrito na sola na fase de apoio do pé durante a marcha); 3) assistência médica e de outros profissionais de saúde disponível para avaliação clínica periódica dos pacientes em risco.

\section{Considerações Finais}

O DM é uma doença sistêmica com sérias manifestações nos membros inferiores, afetando principalmente os pés e causando elevada morbidade e mortalidade para os pacientes. Pé diabético é um termo consagrado que corresponde verdadeiramente a uma síndrome que se apresenta com um largo espectro de sinais e sintomas, todos decorrentes das complicações crônicas e tardias do DM. As manifestações principais do pé diabético são: a neuroartropatia, a ulceração, e a infecção. Estes problemas costumam sobrepor-se às deformidades previamente instaladas, tais como dedos em garra, contratura em equino do pé e distúrbios cutâneos provocados pelo ressecamento da pele. A etiologia destas complicações é multifatorial e inclui: neuropatia, vasculopatia, imunodeficiência e inadequado controle glicêmico.

O adequado manejo dos problemas que afetam os pés nos pacientes diabéticos começa com uma adequada avaliação clínica para permitir o início precoce do tratamento. A ênfase principal deve ser focada nas estratégias de prevenção, destacando-se: 1) educação dos pacientes; 2) monitorização frequente com exames periódicos; 3 ) comunicação direta e compreensível entre o paciente e a equipe multidisciplinar envolvida no tratamento e composta por cirurgiões, clínicos, endocrinologistas, infectologistas, além de cirurgiões ortopédicos especialistas no tratamento das afecções do pé e do tornozelo.

Conflitos de Interesses

O autor declara não haver conflitos de interesses.

\section{Referências}

1 Centers for Disease Control and Prevention. National Diabetics Statistics Report 2017. Department of Health and Human Services, Center for Disease Prevention and Control; 2017

2 Hu FB. Globalization of diabetes: the role of diet, lifestyle, and genes. Diabetes Care 2011;34(06):1249-1257

3 Brodsky JW. The diabetic foot. In: Mann RA, Coughlin MJ, editors. Surgery of the Foot and Ankle. $6^{\text {th }}$ ed. St Louis, MO: Mosby; 1993: 278-283

4 Amaral AH Junior, Amaral LAH, Bastos MG, Nascimento LC, Alves MJ, Andrade MAP. Prevenção das lesões de membros inferiores e redução da morbidade em pacientes diabéticos. Rev Bras Ortop 2014;49(05):482-487

5 Abbott CA, Carrington AL, Ashe H, et al. North-West Diabetes Foot Care Study. The North-West Diabetes Foot Care Study: incidence of, and risk factors for, new diabetic foot ulceration in a community-based patient cohort. Diabet Med 2002;19(05): 377-384

6 Prompers L, Huijberts M, Apelqvist J, et al. High prevalence of ischaemia, infection and serious comorbidity in patients with diabetic foot disease in Europe. Baseline results from the Eurodiale study. Diabetologia 2007;50(01):18-25
7 Prompers L, Schaper N, Apelqvist J, et al. Prediction of outcome in individuals with diabetic foot ulcers: focus on the differences between individuals with and without peripheral arterial disease. The EURODIALE Study. Diabetologia 2008;51(05):747-755

8 Wukich DK, Raspovic KM, Suder NC. Patients with diabetic foot disease fear major lower-extremity amputation more than death. Foot Ankle Spec 2018;11(01):17-21

9 Moulik PK, Mtonga R, Gill GV. Amputation and mortality in newonset diabetic foot ulcers stratified by etiology. Diabetes Care 2003;26(02):491-494

10 Boulton AJ, Vileikyte L, Ragnarson-Tennvall G, Apelqvist J. The global burden of diabetic foot disease. Lancet 2005;366 (9498):1719-1724

11 Cavanagh P, Attinger C, Abbas Z, Bal A, Rojas N, Xu ZR. Cost of treating diabetic foot ulcers in five different countries. Diabetes Metab Res Rev 2012;28(Suppl 1):107-111

12 Gibbons GW, Habershaw GM. Diabetic foot infections. Anatomy and surgery. Infect Dis Clin North Am 1995;9(01):131-142

13 Del Core MA, Ahn J, Lewis RB, et al. The evaluation and treatment of diabetic foot ulcers and diabetic foot infections. Foot Ankle Int. 2018;3(1S):13S-23S

14 Robinson AH, Pasapula C, Brodsky JW. Surgical aspects of the diabetic foot. J Bone Joint Surg Br 2009;91(01):1-7

15 Wukich DK, Shen W, Raspovic KM, Suder NC, Baril DT, Avgerinos E. Noninvasive arterial testing in patients with diabetes: a guide for foot and ankle surgeons. Foot Ankle Int 2015;36(12): 1391-1399

16 Wukich DK, Sadoskas D, Vaudreuil NJ, Fourman M. Comparision of diabetic Charcot patients with and without foot wounds. Foot Ankle Int 2017;38(02):140-148

17 Wukich DK, Raspovic KM, Suder NC. Prevalence of peripheral arterial disease in patients with diabetes Charcot neuroarthropathy. J Foot Ankle Surg 2016;55(04):727-731

18 Richard C, Wadowski M, Goruk S, Cameron L, Sharma AM, Field CJ. Individuals with obesity and type 2 diabetes have additional immune dysfunction compared with obese individuals who are metabolically healthy. BMJ Open Diabetes Res Care 2017;5(01): e000379

19 Grayson ML, Gibbons GW, Balogh K, Levin E, Karchmer AW. Probing to bone in infected pedal ulcers. A clinical sign of underlying osteomyelitis in diabetic patients. JAMA 1995;273 (09):721-723

20 Suder NC, Wukich DK. Prevalence of diabetic neuropathy in patients undergoing foot and ankle surgery. Foot Ankle Spec 2012;5(02):97-101

21 Peterson N, Widnall J, Evans P, Jackson G, Platt S. Diagnostic imaging of diabetic foot disorders. Foot Ankle Int 2017;38(01): 86-95

22 Reiber GE. The epidemiology of diabetic foot problems. Diabet Med 1996;13(uppl 1, Suppl 1)S6-S11

23 Lavery LA, Armstrong DG, Wunderlich RP, Mohler MJ, Wendel CS, Lipsky BA. Risk factors for foot infections in individuals with diabetes. Diabetes Care 2006;29(06):1288-1293

24 Richard JL, Lavigne JP, Sotto A. Diabetes and foot infection: more than double trouble. Diabetes Metab Res Rev 2012;28 (Suppl 1):46-53

25 Lavery LA, Armstrong DG, Harkless LB. Classification of diabetic foot wounds. J Foot Ankle Surg 1996;35(06):528-531

26 Oyibo SO, Jude EB, Tarawneh I, Nguyen HC, Harkless LB, Boulton AJ. A comparison of two diabetic foot ulcer classification systems: the Wagner and the University of Texas wound classification systems. Diabetes Care 2001;24(01):84-88

27 Rossi WR, Rossi FR, Fonseca Filho FF. Pé diabético: o tratamento das úlceras plantares com gesso de contato total e análise dos fatores que interferem no tempo de cicatrização. Rev Bras Ortop 2005;40(03):89-97

28 Sheehan P, Jones P, Caselli A, Giurini JM, Veves A. Percent change in wound area of diabetic foot ulcers over a 4 -week period is a robust 
predictor of complete healing in a 12 -week prospective trial. Diabetes Care 2003;26(06):1879-1882

29 Lavery LA, Higgins KR, La Fontaine J, Zamorano RG, Constantinides GP, Kim PJ. Randomised clinical trial to compare total contact casts, healing sandals and a shear-reducing removable boot to heal diabetic foot ulcers. Int Wound J 2015;12(06):710-715

30 Armstrong DG, Lavery LA, Kimbriel HR, Nixon BP, Boulton AJ. Activity patterns of patients with diabetic foot ulceration: patients with active ulceration may not adhere to a standard pressure off-loading regimen. Diabetes Care 2003;26(09): 2595-2597

31 Mueller MJ, Diamond JE, Sinacore DR, et al. Total contact casting in treatment of diabetic plantar ulcers. Controlled clinical trial. Diabetes Care 1989;12(06):384-388

32 Blume PA, Walters J, Payne W, Ayala J, Lantis J. Comparison of negative pressure wound therapy using vacuum-assisted closure with advanced moist wound therapy in the treatment of diabetic foot ulcers: a multicenter randomized controlled trial. Diabetes Care 2008;31(04):631-636

33 Fedorko L, Bowen JM, Jones W, et al. Hyperbaric oxygen therapy does not reduce indication for amputation in patients with diabetes with nonhealing ulcers of the lower limb: a prospective, double blind, randomized controlled clinical trial. Diabetes Care 2016;39(03):392-399

34 Löndahl M, Katzman P, Nilsson A, Hammarlund C. Hyperbaric oxygen therapy facilitates healing of chronic foot ulcers in patients with diabetes. Diabetes Care 2010;33(05):998-1003

35 Bus SA, van Deursen RW, Armstrong DG, Lewis JE, Caravaggi CF, Cavanagh PR; International Working Group on the Diabetic Foot. Footwear and offloading interventions to prevent and heal foot ulcers and reduce plantar pressure in patients with diabetes: a systematic review. Diabetes Metab Res Rev 2016;32(Suppl 1): 99-118

36 Bus SA, van Netten JJ, Lavery LA, et al. International Working Group on the Diabetic Foot. IWGDF guidance on the prevention of foot ulcers in at-risk patients with diabetes. Diabetes Metab Res Rev 2016;32(Suppl 1):16-24

37 Pound N, Chipchase S, Treece K, Game F, Jeffcoate W. Ulcer-free survival following management of foot ulcers in diabetes. Diabet Med 2005;22(10):1306-1309

38 Reiber GE, Lipsky BA, Gibbons GW. The burden of diabetic foot ulcers. Am J Surg 1998;176(2A, Suppl)5S-10S

39 Taylor SM, Johnson BL, Samies NL, et al. Contemporary management of diabetic neuropathic foot ulceration: a study of 917 consecutively treated limbs. J Am Coll Surg 2011;212(04):532-545 , discussion $546-548$

40 Laborde JM. Neuropathic plantar forefoot ulcers treated with tendon lengthenings. Foot Ankle Int 2008;29(04):378-384

41 Reiber GE, Boyko EJ, Smith DJ. Lower extremity foot ulcers and amputation in diabetes. In: Harris MI, Cowie CC, Stern MP, et al. editors. Diabetes in America. $2^{\text {nd }}$ ed. Bethesda, Md: National Institutes of Health, National Institute of Diabetes and Digestive and Kidney Diseases; 1995:409-428

42 Raspovic KM, Wukich DK. Self-reported quality of life and diabetic foot infections. J Foot Ankle Surg 2014;53(06):716-719
43 Bagdade JD, Root RK, Bulger RJ. Impaired leukocyte function in patients with poorly controlled diabetes. Diabetes 1974;23 (01):9-15

44 Wukich DK, Hobizal KB, Raspovic KM, Rosario BL. SIRS is valid in discriminating between severe and moderate diabetic foot infections. Diabetes Care 2013;36(11):3706-3711

45 Lam K, van Asten SA, Nguyen T, La Fontaine J, Lavery LA. Diagnostic accuracy of probe to bone to detect osteomyelitis in diabetic foot: a systematic review. Clin Infect Dis 2016;63(07):944-948

46 Aragón-Sánchez FJ, Cabrera-Galván JJ, Quintana-Marrero Y, et al. Outcomes of surgical treatment of diabetic foot osteomyelitis: a series of 185 patients with histopathological confirmation of bone involvement. Diabetologia 2008;51(11):1962-1970

47 Lavery LA, Armstrong DG, Peters EJ, Lipsky BA. Probe-to-bone test for diagnosing diabetic foot osteomyelitis: reliable or relic? Diabetes Care 2007;30(02):270-274

48 Morales Lozano R, González Fernández ML, Martinez Hernández D, Beneit Montesinos JV, Guisado Jiménez S, Gonzalez Jurado MA. Validating the probe-to-bone test and other tests for diagnosing chronic osteomyelitis in the diabetic foot. Diabetes Care 2010;33 (10):2140-2145

49 Brodsky JW. Outpatient diagnosis and care of the diabetic foot. Instr Course Lect 1993;42:121-139

50 Cierny G III, Mader JT, Penninck JJ. A clinical staging system for adult osteomyelitis. Clin Orthop Relat Res 2003;(414):7-24

51 Malhotra R, Chan CS, Nather A. Osteomyelitis in the diabetic foot. Diabet Foot Ankle 2014;5:10.3402/dfa.v5.24445

52 Sanverdi SE, Ergen BF, Oznur A. Current challenges in imaging of the diabetic foot. Diabet Foot Ankle 2012;3:10.3402/dfa.v3i0. 18754

53 Lepäntalo M, Apelqvist J, Setacci C, et al. Chapter V: Diabetic foot. Eur J Vasc Endovasc Surg 2011;42(Suppl 2):S60-S74

54 Pineda C, Espinosa R, Pena A. Radiographic imaging in osteomyelitis: the role of plain radiography, computed tomography, ultrasonography, magnetic resonance imaging, and scintigraphy. Semin Plast Surg 2009;23(02):80-89

55 Tone A, Nguyen S, Devemy F, et al. Six-week versus twelve-week antibiotic therapy for nonsurgically treated diabetic foot osteomyelitis: a multicenter open-label controlled randomized study. Diabetes Care 2015;38(02):302-307

56 Schneekloth BJ, Lowery NJ, Wukich DK. Charcot neuroarthropathy in patients with diabetes: an update systematic review of surgical management. J Foot Ankle Surg 2016;55(03):586-590

57 van Battum P, Schaper N, Prompers L, et al. Differences in minor amputation rate in diabetic foot disease throughout Europe are in part explained by differences in disease severity at presentation. Diabet Med 2011;28(02):199-205

58 Wukich DK, Hobizal KB, Brooks MM. Severity of diabetic foot infection and rate of limb salvage. Foot Ankle Int 2013;34(03): 351-358

59 Wukich DK, Ahn J, Raspovic KM, La Fontaine J, Lavery LA. Improved quality of life after transtibial amputations in patients with diabetes-related foot complications. Int J Low Extrem Wounds 2017;16(02):114-121 